Gazi University
Journal of Science
http://dergipark.gov.tr/gujs

\title{
The Modified Modal Operators over the Generalized Interval Valued Intuitionistic Fuzzy Sets
}

\author{
Ezzatallah BALOUI JAMKHANEH \\ Department of Statistics, Qaemshahr Branch, Islamic Azad University, Qaemshahr, Iran
}

\author{
Highlights \\ - The paper focuses on define newly modal operators over GIVIFSs. \\ - Various properties of these operators have also been investigated in details. \\ - Some applications of operators are one motivation for study. \\ - The validity of the operators is tested based on proofs and numerical examples.
}

\begin{tabular}{|c|}
\hline Article Info \\
\hline $\begin{array}{l}\text { Received:19/06/2018 } \\
\text { Accepted:07/12/2018 }\end{array}$ \\
\hline Keywords \\
\hline $\begin{array}{l}\text { Generalized interval } \\
\text { valued intuitionistic } \\
\text { fuzzy sets } \\
\text { Intuitionistic fuzzy sets } \\
\text { Modified modal } \\
\text { operators }\end{array}$ \\
\hline
\end{tabular}

\section{INTRODUCTION}

Atanassov [1] introduced the concept of intuitionistic fuzzy sets (IFSs), which is a generalization of Zadeh's fuzzy sets [2] and defined new operations on IFSs. In IFSs, each element is assigned by membership and non-membership degrees, where the sum of the two degrees is between zero and one. However, in reality, it may not always be true that the degree of membership and degree of non-membership of an element in IFS be real numbers. Therefore, a generalization of IFS was introduced by Atanassov and Gargov [3] as interval valued intuitionistic fuzzy sets (IVIFSs) which its fundamental characteristic is that the values of its membership and non-membership degree are intervals rather than exact numbers. After the introduction of IVIFSs, many researchers have shown interest in the IVIFSs theory and applied it to the various field. Interval valued intuitionistic fuzzy sets is used to model uncertainty, imprecise, incomplete and vague information. Atanassov [4] introduced operators over IVIFSs. Xu [5], Xu and Jian [6] and Wei and Wang [7] developed some arithmetic aggregation operators and some geometric aggregation operators of IVIFS for decision making. Wang and Liu [8] considered the interval valued intuitionistic fuzzy hybrid weighted averaging operator based on Einstein operation and its application to decision making. They defined generalized interval valued intuitionistic fuzzy relation with some results. Bhowmik and Pal $[9,10]$ defined generalized interval valued intuitionistic fuzzy sets (GIVIFSs). Bhowmik and Pal [11] defined two composite relations, four types of reflexivity and irreflexivity of GIVIFSs with some of their properties. Also they define two operators $\mathrm{C}$ and I with some properties over GIVIFSs. 
Li [12-14], Yue [15], Chen et al. [16], Bai [17] and Wang and Chen [18] presented methods for handling multi-criteria fuzzy decision making based on IVIFS. Mondal and Samanta [19] studied the topological properties and the category of topological spaces of IVIFSs. Zhang et al. [20] introduced a generalized interval valued intuitionistic fuzzy sets. Sudharsan and Ezhilmaran [21] defined two new operators over interval valued intuitionistic fuzzy sets. A novel way introduced to fuse several images using interval valued intuitionistic fuzzy sets by Ananthi and Balasubramaniam [22]. They prove that IVIFSs are more suitable for fusion of such uncertain images. Meng et al. [23] analyzed a method to multi-attribute decision making with interval valued intuitionistic fuzzy information problems using prospect theory based on the interval valued intuitionistic hybrid weight averaging operator. Reiser and Bedregal [24] studies the conjugate functions related to main connectives of the interval valued intuitionistic fuzzy logic.

One motivation for our study has been the significant performance achieved by the use of operators over IVIFSs implications in some applications. For example, some applications of operators have been: medical diagnosis (Ahn et al. [25], Ezhilmaran and Sudharsan [26]); decision making problem (Bhowmik and Pal [11]); exploitation investment evaluation (Qi et al. [27]); mathematical programming (Wang et al. [28]); evaluation about the performance of e-government (Zhang et al. [29]); multiple attribute group decision making (Tan et al. [30]); supplier selection with multi criteria group decision making (Makui et al. [31]); medical diagnosis using logical operators (Pathinathan et al. [32]); enterprise e-marketing performance evaluation (Zhou, [33]); etc.

Baloui Jamkhaneh and Nadarajah [34] considered a generalized intuitionistic fuzzy sets $\left(\mathrm{GIFS}_{\mathrm{B}} S\right)$ and introduced some operators over $\mathrm{GIFS}_{\mathrm{B}}$. Afterwards, level operators, modal-like operators, modal operators and some operations were introduced on $\mathrm{GIFS}_{\mathrm{B}} s$ in Baloui Jamkhaneh [35], Baloui Jamkhaneh and Nadi Ghara [36], Baloui Jamkhaneh and Nadarajah [37], Baloui Jamkhaneh and Garg [38]. Baloui Jamkhaneh [39] considered generalized interval valued intuitionistic fuzzy sets $\left(\mathrm{GIVIFS}_{\mathrm{B}} \mathrm{S}\right)$, dealing with uncertainty and vagueness. Afterwards, some operations were introduced on $\mathrm{GIVIFS}_{\mathrm{B}} S$ in Baloui Jamkhaneh [40]. Recently Baloui Jamkhaneh [41] and Baloui Jamkhaneh and Amirzadi [42] defined some operators over GIVIFS $_{B} s$ due to Baloui Jamkhaneh [39]. According to the definition in Baloui Jamkhaneh [39], degree of membership and degree of non-membership of GIVIFS $_{B}$ are subintervals of the interval $[0,1]$. In order to establish this condition for operators due to Baloui Jamkhaneh [41], the $\alpha$ and $\beta$ parameters must be in the specific subset of $[0,1]$. This means that the values of the parameters must be limited. In this case, this reduces the integrity and comprehensiveness of the operator. For this purpose, in this paper, modified operators are defined in which parameters are not limited.

In this paper we shall introduce the some of the modified modal operators (as $D_{\alpha}(A), F_{\alpha, \beta}(A)$, $\left.\mathrm{J}_{\alpha, \beta}(A), \mathrm{d}_{\alpha}(A), \mathrm{f}_{\alpha, \beta}(A), \mathrm{j}_{\alpha, \beta}(A), \mathrm{H}_{\alpha, \beta}(A), \mathrm{h}_{\alpha, \beta}(A)\right)$ over $\operatorname{GIVIFS}_{B}$ and we will discuss their properties. Some of these properties are the following: i) All these operators are GIVIFS ${ }_{B}$ ii) All these operators are increasing relative to $\alpha$ iii) All these operators are decreasing relative to $\beta$ iv) $D_{0}(A)=F_{0,1}(A)=\overline{d_{1}(A)}=$ $\overline{\mathrm{f}_{1,0}(\mathrm{~A})}=\mathrm{H}_{1,1}(\mathrm{~A})=\overline{\mathrm{J}_{1,1}(\mathrm{~A})}=\square \mathrm{A} \quad$ v) $\mathrm{D}_{1}(\mathrm{~A})=\mathrm{F}_{1,0}(\mathrm{~A})=\mathrm{J}_{1,1}(\mathrm{~A})=\overline{\mathrm{d}_{0}(\mathrm{~A})}=\overline{\mathrm{f}_{0,1}(\mathrm{~A})}=\overline{\mathrm{h}_{1,1}(\mathrm{~A})}=\checkmark \mathrm{A}$ vi) $A=\mathrm{F}_{0,0}(A)=\mathrm{J}_{0,1}(A)=\mathrm{H}_{1,0}(A) \quad$ vii) $\bar{A}=\mathrm{f}_{0,0}(A)=\mathrm{j}_{0,1}(A)=\mathrm{h}_{1,0}(A)$ viii) $A \subset \mathrm{J}_{\alpha, \beta}(A), \bar{A} \subset$ $\mathrm{j}_{\alpha, \beta}(\mathrm{A}) \quad$ ix) $\left.\quad \mathrm{D}_{\alpha}(\square \mathrm{A})=\mathrm{F}_{\alpha, \beta}(\square \mathrm{A})=\overline{\mathrm{d}_{\alpha}(\square \mathrm{A})}=\overline{\mathrm{f}_{\alpha, \beta}(\square \mathrm{A})}=\square \mathrm{A} \quad \mathrm{x}\right) \mathrm{D}_{\alpha}(\diamond \mathrm{A})=\mathrm{F}_{\alpha, \beta}(\diamond \mathrm{A})=\overline{\mathrm{d}_{\alpha}(\nabla \mathrm{A})}=$ $\overline{f_{\alpha, \beta}(\nabla A)}=\diamond A$, etc. The remainder of the paper is organized as follows. In Section 2, we briefly introduce IFS and its generalizations. In Section 3 define modified operators over generalized interval valued intuitionistic fuzzy sets. The paper is concluded in Section 4.

\section{REMARKS ON THE GIVIFS}

In this section, we give some basic definition. Let $\mathrm{X}$ be a non-empty universal set.

Definition 2.1. [1] An IFS $A$ in $X$ is defined as an object of the form $A=\left\{\left\langle x, \mu_{A}(x), v_{A}(x)\right\rangle: x \in X\right\}$ where the functions $\mu_{\mathrm{A}}: \mathrm{X} \rightarrow[0,1]$ and $v_{\mathrm{A}}: \mathrm{X} \rightarrow[0,1]$ denote the degree of membership and degree of nonmembership of the element $\mathrm{x}$ in A respectively, satisfying $0 \leq \mu_{A}(\mathrm{x})+v_{A}(\mathrm{x}) \leq 1$ for each $\mathrm{x} \in \mathrm{X}$. 
Definition 2.2. Let [I] be the set of all closed subintervals of the interval $[0,1]$ and $M_{A}(x)=$ $\left[\mathrm{M}_{\mathrm{AL}}(\mathrm{x}), \mathrm{M}_{\mathrm{AU}}(\mathrm{x})\right] \in[\mathrm{I}]$ and $\mathrm{N}_{\mathrm{A}}(\mathrm{x})=\left[\mathrm{N}_{\mathrm{AL}}(\mathrm{x}), \mathrm{N}_{\mathrm{AU}}(\mathrm{x})\right] \in[\mathrm{I}]$ then $\mathrm{N}_{\mathrm{A}}(\mathrm{x}) \leq \mathrm{M}_{\mathrm{A}}(\mathrm{x})$ if and only if $\mathrm{N}_{\mathrm{AL}}(\mathrm{x}) \leq \mathrm{M}_{\mathrm{AL}}(\mathrm{x})$ and $\mathrm{N}_{\mathrm{AU}}(\mathrm{x}) \leq \mathrm{M}_{\mathrm{AU}}(\mathrm{x})$.

Definition 2.3. [3] Interval valued intuitionistic fuzzy set (IVIFS) $A$ in $X$, is defined as an object of the form $A=\left\{\left\langle x, M_{A}(x), N_{A}(x)\right\rangle: x \in X\right\}$ where the functions $M_{A}(x): X \rightarrow[I]$ and $N_{A}(x): X \rightarrow[I]$, denote the degree of membership and degree of non-membership of the element $x$ in A respectively, where $M_{A}(x)=$ $\left[\mathrm{M}_{\mathrm{AL}}(\mathrm{x}), \mathrm{M}_{\mathrm{AU}}(\mathrm{x})\right], \mathrm{N}_{\mathrm{A}}(\mathrm{x})=\left[\mathrm{N}_{\mathrm{AL}}(\mathrm{x}), \mathrm{N}_{\mathrm{AU}}(\mathrm{x})\right]$, and $0 \leq \mathrm{M}_{\mathrm{AU}}(\mathrm{x})+\mathrm{N}_{\mathrm{AU}}(\mathrm{x}) \leq 1$ for each $\mathrm{x} \in \mathrm{X}$.

Definition 2.4. [34] Let $X$ be a non-empty set. Generalized intuitionistic fuzzy set $A$ in $X$, is defined as an object of the form $A=\left\{\left\langle\mathrm{X}, \mu_{\mathrm{A}}(\mathrm{x}), v_{\mathrm{A}}(\mathrm{x})\right\rangle: \mathrm{x} \in \mathrm{X}\right\}$ where the functions $\mu_{\mathrm{A}}: \mathrm{X} \rightarrow[0,1]$ and $v_{\mathrm{A}}: \mathrm{X} \rightarrow[0,1]$, denote the degree of membership and degree of non-membership of the element $\mathrm{x}$ in A respectively, and $0 \leq \mu_{\mathrm{A}}(\mathrm{x})^{\delta}+\mathrm{v}_{\mathrm{A}}(\mathrm{x})^{\delta} \leq 1$ for each $\mathrm{x} \in \mathrm{X}$, and $\delta=\mathrm{n}$ or $\frac{1}{\mathrm{n}}, \mathrm{n}=1,2, \ldots, \mathrm{N}$.

Definition 2.5. [39] Generalized interval valued intuitionistic fuzzy set $\left(\operatorname{GIVIFS}_{B}\right) A$ in $X$, is defined as an object of the form $A=\left\{\left\langle x, M_{A}(x), N_{A}(x)\right\rangle: x \in X\right\}$ where the functions $M_{A}(x): X \rightarrow[I]$ and $N_{A}(x): X \rightarrow$ [I], denote the degree of membership and degree of non-membership of the element $x$ in A respectively, and $\mathrm{M}_{\mathrm{A}}(\mathrm{x})=\left[\mathrm{M}_{\mathrm{AL}}(\mathrm{x}), \mathrm{M}_{\mathrm{AU}}(\mathrm{x})\right], \mathrm{N}_{\mathrm{A}}(\mathrm{x})=\left[\mathrm{N}_{\mathrm{AL}}(\mathrm{x}), \mathrm{N}_{\mathrm{AU}}(\mathrm{x})\right]$, where $0 \leq \mathrm{M}_{\mathrm{AU}}(\mathrm{x})^{\delta}+\mathrm{N}_{\mathrm{AU}}(\mathrm{x})^{\delta} \leq$ 1 , for each $\mathrm{x} \in \mathrm{X}$ and $\delta=\mathrm{n}$ or $\frac{1}{\mathrm{n}}, \mathrm{n}=1,2, \ldots, \mathrm{N}$. The collection of all $\operatorname{GIVIFS}_{\mathrm{B}}(\delta)$ is denoted by $\operatorname{GIVIFS}_{\mathrm{B}}(\delta, \mathrm{X})$.

Definition 2.6. The degree of non-determinacy (uncertainty) of an element $x \in X$ to the $\operatorname{GIVIFS}_{B} A$ is defined by

$\pi_{\mathrm{A}}(\mathrm{x})=\left[\pi_{\mathrm{AL}}(\mathrm{x}), \pi_{\mathrm{AU}}(\mathrm{x})\right]=\left[\left(1-\mathrm{M}_{\mathrm{AU}}(\mathrm{x})^{\delta}-\mathrm{N}_{\mathrm{AU}}(\mathrm{x})^{\delta}\right)^{\frac{1}{\delta}},\left(1-\mathrm{M}_{\mathrm{AL}}(\mathrm{x})^{\delta}-\mathrm{N}_{\mathrm{AL}}(\mathrm{x})^{\delta}\right)^{\frac{1}{\delta}}\right]$.

Definition 2.7. [39] Let A and B be two GIVIFS ${ }_{B} S$ such that

$$
\begin{array}{ll}
A=\left\{\left\langle x, M_{A}(x), N_{A}(x)\right\rangle: x \in X\right\}, \quad B=\left\{\left\langle x, M_{B}(x), N_{B}(x)\right\rangle: x \in X\right\} \\
M_{A}(x)=\left[M_{A L}(x), M_{A U}(x)\right], & N_{A}(x)=\left[N_{A L}(x), N_{A U}(x)\right] \\
M_{B}(x)=\left[M_{B L}(x), M_{B U}(x)\right], & N_{B}(x)=\left[N_{B L}(x), N_{B U}(x)\right]
\end{array}
$$

Define the following relations on A and B

$$
\begin{array}{cl}
\text { i. } & A \subset B \text { if and only if } M_{A}(x) \leq M_{B}(x) \text { and } N_{A}(x) \geq N_{B}(x), \forall x \in X, \\
\text { ii. } & A \subset_{\square} B \text { if and only if } M_{A}(x) \leq M_{B}(x), \forall x \in X, \\
\text { iii. } & A \subset b \text { if and only if } N_{A}(x) \geq N_{B}(x), \forall x \in X, \\
\text { iv. } & A \cup B=\left\{\left\langlex,\left[\max \left(M_{A L}(x), M_{B L}(x)\right), \max \left(M_{A U}(x), M_{B U}(x)\right)\right],\right.\right. \\
& \left.\left.\left[\min \left(N_{A L}(x), N_{B L}(x)\right), \min \left(N_{A U}(x), N_{B U}(x)\right)\right]\right\rangle: x \in X\right\}, \\
\text { v. } & A \cap B=\left\{\left\langlex,\left[\min \left(M_{A L}(x), M_{B L}(x)\right), \min \left(M_{A U}(x), M_{B U}(x)\right)\right],\right.\right. \\
& \left.\quad\left[\max \left(N_{A L}(x), N_{B L}(x)\right), \max \left(N_{A U}(x), N_{B U}(x)\right]\right\rangle: x \in X\right\}, \\
\text { vi. } & \bar{A}=\left\{\left\langle x, N_{A}(x), M_{A}(x)\right\rangle: x \in X\right\} .
\end{array}
$$

Definition 2.8. [42] For every $\operatorname{GIVIFS}_{B} A=\left\{\left\langle x, M_{A}(x), N_{A}(x)\right\rangle: x \in X\right\}$, the modal logic operators defined as follows

The Necessity measure on A:

$$
\square A=\left\{\left\langle x,\left[M_{A L}(x), M_{A U}(x)\right],\left[N_{A L}(x),\left(1-M_{A U}(x)^{\delta}\right)^{\frac{1}{\delta}}\right]\right\rangle: x \in X\right\}
$$

The Possibility measure on A: 


$$
\Delta \mathrm{A}=\left\{\left\langle\mathrm{x},\left[\mathrm{M}_{\mathrm{AL}}(\mathrm{x}),\left(1-\mathrm{N}_{\mathrm{AU}}(\mathrm{x})^{\delta}\right)^{\frac{1}{\delta}}\right],\left[\mathrm{N}_{\mathrm{AL}}(\mathrm{x}), \mathrm{N}_{\mathrm{AU}}(\mathrm{x})\right]\right\rangle: \mathrm{x} \in \mathrm{X}\right\}
$$

\section{THE MODIFIED MODAL OPERATORS OF GIVIFS}

Here, we will introduce new operators over the GIVIFS $_{B}$, which modified some operators due to Baloui Jamkhaneh [41] related to GIVIFS ${ }_{B}$. Let $X$ is a non-empty finite set and $A=\left\{\left\langle x, M_{A}(x), N_{A}(x)\right\rangle: x \in X\right\}$ is a GIVIFS $_{B}$.

Definition 3.1. Let $A \in$ GIVIFS $_{B}$ and $\alpha \in[0,1]$, we define the operator of $D_{\alpha}(A, \delta)$ (in summary, we will show as $\left.\mathrm{D}_{\alpha}(\mathrm{A})\right)$ as follows

$\mathrm{D}_{\alpha}(\mathrm{A})=\left\{\left\langle\mathrm{x}, \mathrm{M}_{\mathrm{D}_{\alpha}}(\mathrm{A}), \mathrm{N}_{\mathrm{D}_{\alpha}}(\mathrm{A})\right\rangle: \mathrm{x} \in \mathrm{X}\right\}$,

$\mathrm{M}_{\mathrm{D}_{\alpha}}(\mathrm{A})=\left[\mathrm{M}_{\mathrm{AL}}(\mathrm{x}),\left(\mathrm{M}_{\mathrm{AU}}(\mathrm{x})^{\delta}+\alpha \pi_{\mathrm{AL}}(\mathrm{x})^{\delta}\right)^{\frac{1}{\delta}}\right], \mathrm{N}_{\mathrm{D}_{\alpha}}(\mathrm{A})=\left[\mathrm{N}_{\mathrm{AL}}(\mathrm{x}),\left(\mathrm{N}_{\mathrm{AU}}(\mathrm{x})^{\delta}+(1-\alpha) \pi_{\mathrm{AL}}(\mathrm{x})^{\delta}\right)^{\frac{1}{\delta}}\right]$.

It can be easily shown that $\pi_{\mathrm{D}_{\alpha}(\mathrm{A}) \mathrm{L}}(\mathrm{x})=0$.

Theorem 3.1. For every A $\in \mathrm{GIVIFS}_{\mathrm{B}}$ and $\alpha \in[0,1]$, it holds that

$$
\begin{aligned}
\text { i. } & D_{\alpha}(A) \in \operatorname{GIVIFS}_{B}, \\
\text { ii. } & D_{0}(A)=\square A, \\
\text { iii. } & D_{1}(A)=\diamond A, \\
\text { iv. } & D_{\alpha}(\bar{A})=\overline{D_{1-\alpha}(A)} \\
\text { v. } & D_{\alpha}\left(D_{\alpha}(A)\right)=D_{\alpha}(A) .
\end{aligned}
$$

Proof. The proof of part (i) is straightforward.

(ii) Note that

$$
\begin{aligned}
\mathrm{M}_{\mathrm{D}_{0}}(\mathrm{~A}) & =\left[\mathrm{M}_{\mathrm{AL}}(\mathrm{x}),\left(\mathrm{M}_{\mathrm{AU}}(\mathrm{x})^{\delta}+0 \times \pi_{\mathrm{AL}}(\mathrm{x})^{\delta}\right)^{\frac{1}{\delta}}\right] \\
& =\left[\mathrm{M}_{\mathrm{AL}}(\mathrm{x}), \mathrm{M}_{\mathrm{AU}}(\mathrm{x})\right], \\
\mathrm{N}_{\mathrm{D}_{0}}(\mathrm{~A}) & =\left[\mathrm{N}_{\mathrm{AL}}(\mathrm{x}),\left(\mathrm{N}_{\mathrm{AU}}(\mathrm{x})^{\delta}+(1-0) \pi_{\mathrm{AL}}(\mathrm{x})^{\delta}\right)^{\frac{1}{\delta}}\right] \\
& =\left[\mathrm{N}_{\mathrm{AL}}(\mathrm{x}),\left(\mathrm{N}_{\mathrm{AU}}(\mathrm{x})^{\delta}+\pi_{\mathrm{AL}}(\mathrm{x})^{\delta}\right)^{\frac{1}{\delta}}\right] .
\end{aligned}
$$

Since $\pi_{\mathrm{AL}}(\mathrm{x})^{\delta}=1-\mathrm{M}_{\mathrm{AU}}(\mathrm{x})^{\delta}-\mathrm{N}_{\mathrm{AU}}(\mathrm{x})^{\delta}$, then $\mathrm{N}_{\mathrm{D}_{0}}(\mathrm{~A})=\left[\mathrm{N}_{\mathrm{AL}}(\mathrm{x}),\left(1-\mathrm{M}_{\mathrm{AU}}(\mathrm{x})^{\delta}\right)^{\frac{1}{\delta}}\right]$, finally we have $\mathrm{D}_{0}(\mathrm{~A})=\left\{\left\langle\mathrm{x},\left[\mathrm{M}_{\mathrm{AL}}(\mathrm{x}), \mathrm{M}_{\mathrm{AU}}(\mathrm{x})\right],\left[\mathrm{N}_{\mathrm{AL}}(\mathrm{x}),\left(1-\mathrm{M}_{\mathrm{AU}}(\mathrm{x})^{\delta}\right)^{\frac{1}{\delta}}\right]\right\rangle: \mathrm{x} \in \mathrm{X}\right\}=\square \mathrm{A}$.

The proof is complete.

(iii) Note that

$$
\begin{aligned}
& D_{1}(A)=\left\{\left\langle x, M_{D_{1}}(A), N_{D_{1}}(A)\right\rangle: x \in X\right\} \\
& M_{D_{1}}(A)=\left[M_{A L}(x),\left(M_{A U}(x)^{\delta}+\pi_{A L}(x)^{\delta}\right)^{\frac{1}{\delta}}\right]
\end{aligned}
$$




$$
\begin{aligned}
& =\left[\mathrm{M}_{\mathrm{AL}}(\mathrm{x}),\left(1-\mathrm{N}_{\mathrm{AU}}(\mathrm{x})^{\delta}\right)^{\frac{1}{\delta}}\right], \\
\mathrm{N}_{\mathrm{D}_{1}}(\mathrm{~A}) & =\left[\mathrm{N}_{\mathrm{AL}}(\mathrm{x}),\left(\mathrm{N}_{\mathrm{AU}}(\mathrm{x})^{\delta}+(1-1) \pi_{\mathrm{AL}}(\mathrm{x})^{\delta}\right)^{\frac{1}{\delta}}\right], \\
& =\left[\mathrm{N}_{\mathrm{AL}}(\mathrm{x}), \mathrm{N}_{\mathrm{AU}}(\mathrm{x})\right],
\end{aligned}
$$

then

$\mathrm{D}_{1}(\mathrm{~A})=\left\{\left\langle\mathrm{x},\left[\mathrm{M}_{\mathrm{AL}}(\mathrm{x}),\left(1-\mathrm{N}_{\mathrm{AU}}(\mathrm{x})^{\delta}\right)^{\frac{1}{\delta}}\right],\left[\mathrm{N}_{\mathrm{AL}}(\mathrm{x}), \mathrm{N}_{\mathrm{AU}}(\mathrm{x})\right]\right\rangle: \mathrm{x} \in \mathrm{X}\right\}=\diamond \mathrm{A}$

The proof is complete. Proofs of (iv) and (v) are obvious.

Definition 3.2. Let $A \in \operatorname{GIVIFS}_{B}$ and $\alpha, \beta \in[0,1]$, where $0 \leq \alpha+\beta \leq 1$, we define the operator of $\mathrm{F}_{\alpha, \beta}(\mathrm{A}, \delta)$ (in summary, we will show as $\mathrm{F}_{\alpha, \beta}(\mathrm{A})$ ) as follows

$$
\begin{aligned}
& \mathrm{F}_{\alpha, \beta}(\mathrm{A})=\left\{\left\langle\mathrm{x}, \mathrm{M}_{\mathrm{F}_{\alpha}}(\mathrm{A}), \mathrm{N}_{\mathrm{F}_{\beta}}(\mathrm{A})\right\rangle: \mathrm{x} \in \mathrm{X}\right\}, \\
& \mathrm{M}_{\mathrm{F}_{\alpha}}(\mathrm{A})=\left[\mathrm{M}_{\mathrm{AL}}(\mathrm{x}),\left(\mathrm{M}_{\mathrm{AU}}(\mathrm{x})^{\delta}+\alpha \pi_{\mathrm{AL}}(\mathrm{x})^{\delta}\right)^{\frac{1}{\delta}}\right], \mathrm{N}_{\mathrm{F}_{\beta}}(\mathrm{A})=\left[\mathrm{N}_{\mathrm{AL}}(\mathrm{x}),\left(\mathrm{N}_{\mathrm{AU}}(\mathrm{x})^{\delta}+\beta \pi_{\mathrm{AL}}(\mathrm{x})^{\delta}\right)^{\frac{1}{\delta}}\right] .
\end{aligned}
$$

Theorem 3.2. For every $A \in$ GIVIFS $_{B}$ and $\alpha, \beta, \gamma \in[0,1]$, where $0 \leq \alpha+\beta \leq 1$, it holds that

$$
\begin{aligned}
\text { i. } & \mathrm{F}_{\alpha, \beta}(\mathrm{A}) \in \mathrm{GIVIFS}_{\mathrm{B}}, \\
\text { ii. } & 0 \leq \gamma \leq \alpha \Rightarrow \mathrm{F}_{\gamma, \beta}(\mathrm{A}) \subset \mathrm{F}_{\alpha, \beta}(\mathrm{A}), \quad 0 \leq \gamma+\beta \leq 1, \\
\text { iii. } & 0 \leq \gamma \leq \beta \Rightarrow \mathrm{F}_{\alpha, \beta}(\mathrm{A}) \subset \mathrm{F}_{\alpha, \gamma}(\mathrm{A}), 0 \leq \alpha+\gamma \leq 1, \\
\text { iv. } & \mathrm{D}_{\alpha}(\mathrm{A})=\mathrm{F}_{\alpha, 1-\alpha}(\mathrm{A}), \\
\text { v. } & \square \mathrm{A}=\mathrm{F}_{0,1}(\mathrm{~A}), \\
\text { vi. } & \nabla \mathrm{A}=\mathrm{F}_{1,0}(\mathrm{~A}), \\
\text { vii. } & \mathrm{F}_{\alpha, \beta}(\overline{\mathrm{A}})=\overline{\mathrm{F}}_{\beta, \alpha}(\mathrm{A}), \\
\text { viii. } & \mathrm{F}_{0,0}(\mathrm{~A})=\mathrm{A}, \\
\text { ix. } & \mathrm{D}_{\alpha}(\mathrm{A}) \subset \mathrm{F}_{\alpha, \beta}(\mathrm{A}) .
\end{aligned}
$$

Proof. (i) Follows since

$$
\begin{aligned}
\mathrm{M}_{\mathrm{F}_{\alpha}(\mathrm{A}) \mathrm{U}}(\mathrm{x})^{\delta} & +\mathrm{N}_{\mathrm{F}_{\beta}(\mathrm{A}) \mathrm{U}}(\mathrm{x})^{\delta} \\
& =\left[\left(\mathrm{M}_{\mathrm{AU}}(\mathrm{x})^{\delta}+\alpha \pi_{\mathrm{AL}}(\mathrm{x})^{\delta}\right)^{\frac{1}{\delta}}\right]^{\delta}+\left[\left(\mathrm{N}_{\mathrm{AU}}(\mathrm{x})^{\delta}+\beta \pi_{\mathrm{AL}}(\mathrm{x})^{\delta}\right)^{\frac{1}{\delta}}\right]^{\delta}, \\
& =\mathrm{M}_{\mathrm{AU}}(\mathrm{x})^{\delta}+\alpha \pi_{\mathrm{AL}}(\mathrm{x})^{\delta}+\mathrm{N}_{\mathrm{AU}}(\mathrm{x})^{\delta}+\beta \pi_{\mathrm{AL}}(\mathrm{x})^{\delta}, \\
& =\mathrm{M}_{\mathrm{AU}}(\mathrm{x})^{\delta}+\mathrm{N}_{\mathrm{AU}}(\mathrm{x})^{\delta}+(\alpha+\beta) \pi_{\mathrm{AL}}(\mathrm{x})^{\delta}, \\
& \leq \mathrm{M}_{\mathrm{AU}}(\mathrm{x})^{\delta}+\mathrm{N}_{\mathrm{AU}}(\mathrm{x})^{\delta}+\pi_{\mathrm{AL}}(\mathrm{x})^{\delta}=1 .
\end{aligned}
$$

Proofs of (ii) and (iii) are obvious.

(iv) Follows since

$$
\mathrm{M}_{\mathrm{F}_{\alpha}}(\mathrm{A})=\left[\mathrm{M}_{\mathrm{AL}}(\mathrm{x}),\left(\mathrm{M}_{\mathrm{AU}}(\mathrm{x})^{\delta}+\alpha \pi_{\mathrm{AL}}(\mathrm{x})^{\delta}\right)^{\frac{1}{\delta}}\right], \mathrm{N}_{\mathrm{F}_{1-\alpha}}(\mathrm{A})=\left[\mathrm{N}_{\mathrm{AL}}(\mathrm{x}),\left(\mathrm{N}_{\mathrm{AU}}(\mathrm{x})^{\delta}+(1-\alpha) \pi_{\mathrm{AL}}(\mathrm{x})^{\delta}\right)^{\frac{1}{\delta}}\right],
$$

then

$$
\mathrm{F}_{\alpha, 1-\alpha}(\mathrm{A})=\left\{\left\langle\mathrm{x}, \mathrm{M}_{\mathrm{F}_{\alpha}}(\mathrm{A}), \mathrm{N}_{\mathrm{F}_{1-\alpha}}(\mathrm{A})\right\rangle: \mathrm{x} \in \mathrm{X}\right\}=\mathrm{D}_{\alpha}(\mathrm{A}) .
$$


(v) Since $D_{0}(A)=F_{0,1}(A)$ by using Theorem3.1 it follows that $F_{0,1}(A)=\square A$.

(vi) Since $D_{1}(A)=F_{1,0}(A)$ by using Theorem3.1 it follows that $F_{1,0}(A)=\diamond A$.

(vii) We have

$$
\begin{aligned}
& \mathrm{F}_{\beta, \alpha}(\mathrm{A})=\left\{\left\langle\mathrm{x}, \mathrm{M}_{\mathrm{F}_{\beta}}(\mathrm{A}), \mathrm{N}_{\mathrm{F}_{\alpha}}(\mathrm{A})\right\rangle: \mathrm{x} \in \mathrm{X}\right\}, \\
& \mathrm{M}_{\mathrm{F}_{\beta}}(\mathrm{A})=\left[\mathrm{M}_{\mathrm{AL}}(\mathrm{x}),\left(\mathrm{M}_{\mathrm{AU}}(\mathrm{x})^{\delta}+\beta \pi_{\mathrm{AL}}(\mathrm{x})^{\delta}\right)^{\frac{1}{\delta}}\right], \mathrm{N}_{\mathrm{F}_{\alpha}}(\mathrm{A})=\left[\mathrm{N}_{\mathrm{AL}}(\mathrm{x}),\left(\mathrm{N}_{\mathrm{AU}}(\mathrm{x})^{\delta}+\alpha \pi_{\mathrm{AL}}(\mathrm{x})^{\delta}\right)^{\frac{1}{\delta}}\right]
\end{aligned}
$$

and

$\mathrm{F}_{\alpha, \beta}(\overline{\mathrm{A}})=\left\{\left\langle\mathrm{x}, \mathrm{M}_{\mathrm{F}_{\alpha}}(\overline{\mathrm{A}}), \mathrm{N}_{\mathrm{F}_{\beta}}(\overline{\mathrm{A}})\right\rangle: \mathrm{x} \in \mathrm{X}\right\}$,

$\mathrm{M}_{\mathrm{F}_{\alpha}}(\overline{\mathrm{A}})=\left[\mathrm{N}_{\mathrm{AL}}(\mathrm{x}),\left(\mathrm{N}_{\mathrm{AU}}(\mathrm{x})^{\delta}+\alpha \pi_{\mathrm{AL}}(\mathrm{x})^{\delta}\right)^{\frac{1}{\delta}}\right], \mathrm{N}_{\mathrm{F}_{\beta}}(\overline{\mathrm{A}})=\left[\mathrm{M}_{\mathrm{AL}}(\mathrm{x}),\left(\mathrm{M}_{\mathrm{AU}}(\mathrm{x})^{\delta}+\beta \pi_{\mathrm{AL}}(\mathrm{x})^{\delta}\right)^{\frac{1}{\delta}}\right]$,

Finally, we have $F_{\alpha, \beta}(\bar{A})=\overline{F_{\beta, \alpha}(A)}$. The proof of part (viii) is straightforward.

(ix) It follows from the fact that $\beta \leq 1-\alpha$.

Definition 3.3. Let $A \in \mathrm{GIVIFS}_{\mathrm{B}}$ and $\alpha, \beta \in[0,1]$, we define the operator of $\mathrm{J}_{\alpha, \beta}(\mathrm{A}, \delta)$ (in summary, we will show as $\left.\mathrm{J}_{\alpha, \beta}(\mathrm{A})\right)$ as follows

$$
\begin{aligned}
& \mathrm{J}_{\alpha, \beta}(\mathrm{A})=\left\{\left\langle\mathrm{x}, \mathrm{M}_{\mathrm{J}_{\alpha, \beta}}(\mathrm{A}), \mathrm{N}_{\mathrm{J}_{\alpha, \beta}}(\mathrm{A})\right\rangle: \mathrm{x} \in \mathrm{X}\right\} \\
& \mathrm{M}_{\mathrm{J}_{\alpha, \beta}}(\mathrm{A})=\left[\mathrm{M}_{\mathrm{AL}}(\mathrm{x}),\left(\mathrm{M}_{\mathrm{AU}}(\mathrm{x})^{\delta}+\alpha \pi_{\mathrm{AL}}(\mathrm{x})^{\delta}\right)^{\frac{1}{\delta}}\right], \mathrm{N}_{\mathrm{J}_{\alpha, \beta}}(\mathrm{A})=\left[\beta^{\frac{1}{\delta}} \mathrm{N}_{\mathrm{AL}}(\mathrm{x}), \beta^{\frac{1}{\delta}} \mathrm{N}_{\mathrm{AU}}(\mathrm{x})\right]
\end{aligned}
$$

Theorem 3.3. For every $A \in \operatorname{GIVIFS}_{B}$ and $\alpha, \beta, \gamma \in[0,1]$, it holds that

$$
\begin{aligned}
\text { i. } & \mathrm{J}_{\alpha, \beta}(\mathrm{A}) \in \mathrm{GIVIFS}_{\mathrm{B}}, \\
\text { ii. } & \alpha \leq \gamma \Rightarrow \mathrm{J}_{\alpha, \beta}(\mathrm{A}) \subset \mathrm{J}_{\gamma, \beta}(\mathrm{A}), \\
\text { iii. } & \beta \leq \gamma \Rightarrow \mathrm{J}_{\alpha, \gamma}(\mathrm{A}) \subset \mathrm{J}_{\alpha, \beta}(\mathrm{A}), \\
\text { iv. } & \quad \mathrm{A}=\mathrm{J}_{1,1}(\mathrm{~A}), \\
\text { v. } & \mathrm{A}=\mathrm{J}_{0,1}(\mathrm{~A}), \\
\text { vi. } & \mathrm{A} \subset \mathrm{J}_{\alpha, \beta}(\mathrm{A}) .
\end{aligned}
$$

Proof. (i) Follows since

$$
\begin{aligned}
\mathrm{M}_{\mathrm{J}_{\alpha, \beta}(\mathrm{A}) \mathrm{U}}(\mathrm{x})^{\delta}+\mathrm{N}_{\mathrm{J}_{\alpha, \beta}(\mathrm{A}) \mathrm{U}}(\mathrm{x})^{\delta} & \left.=\left(\mathrm{M}_{\mathrm{AU}}(\mathrm{x})^{\delta}+\alpha \pi_{\mathrm{AL}}(\mathrm{x})^{\delta}\right)^{\frac{1}{\delta}}\right)^{\delta}+\left(\beta^{\frac{1}{\delta}} \mathrm{N}_{\mathrm{AU}}(\mathrm{x})\right)^{\delta}, \\
& =\left(\mathrm{M}_{\mathrm{AU}}(\mathrm{x})^{\delta}+\alpha \pi_{\mathrm{AL}}(\mathrm{x})^{\delta}\right)+\beta \mathrm{N}_{\mathrm{AU}}(\mathrm{x})^{\delta} \\
& \leq \mathrm{M}_{\mathrm{AU}}(\mathrm{x})^{\delta}+\pi_{\mathrm{AL}}(\mathrm{x})^{\delta}+\mathrm{N}_{\mathrm{AU}}(\mathrm{x})^{\delta}=1 .
\end{aligned}
$$

(ii) Since $\alpha \leq \gamma$ then it is clear that

$$
\left[\mathrm{M}_{\mathrm{AL}}(\mathrm{x}),\left(\mathrm{M}_{\mathrm{AU}}(\mathrm{x})^{\delta}+\alpha \pi_{\mathrm{AL}}(\mathrm{x})^{\delta}\right)^{\frac{1}{\delta}}\right] \leq\left[\mathrm{M}_{\mathrm{AL}}(\mathrm{x}),\left(\mathrm{M}_{\mathrm{AU}}(\mathrm{x})^{\delta}+\gamma \pi_{\mathrm{AL}}(\mathrm{x})^{\delta}\right)^{\frac{1}{\delta}}\right]
$$

Finally we have $J_{\alpha, \beta}(A) \subset J_{\gamma, \beta}(A)$. This completes the proof.

The proof of (iii) is similar to that of (ii). Proofs of (iv), (v) and (vi) are obvious. 
Definition 3.4. Let $\alpha \in[0,1]$ and $A \in \operatorname{GIVIFS}_{B}$, we define the operator of $d_{\alpha}(A, \delta)$ (in summary, we will show as $\left.d_{\alpha}(A)\right)$ as follows

$\mathrm{d}_{\alpha}(\mathrm{A})=\left\{\left\langle\mathrm{x}, \mathrm{M}_{\mathrm{d}_{\alpha}}(\mathrm{A}), \mathrm{N}_{\mathrm{d}_{\alpha}}(\mathrm{A})\right\rangle: \mathrm{x} \in \mathrm{X}\right\}$,
$\mathrm{M}_{\mathrm{d}_{\alpha}}(\mathrm{A})=\left[\mathrm{N}_{\mathrm{AL}}(\mathrm{x}),\left(\mathrm{N}_{\mathrm{AU}}(\mathrm{x})^{\delta}+\alpha \pi_{\mathrm{AL}}(\mathrm{x})^{\delta}\right)^{\frac{1}{\delta}}\right], \mathrm{N}_{\mathrm{d}_{\alpha}}(\mathrm{A})=\left[\mathrm{M}_{\mathrm{AL}}(\mathrm{x}),\left(\mathrm{M}_{\mathrm{AU}}(\mathrm{x})^{\delta}+(1-\alpha) \pi_{\mathrm{AL}}(\mathrm{x})^{\delta}\right)^{\frac{1}{\delta}}\right]$.

It can be easily shown that $\pi_{d_{\alpha}(A) L}(x)=0$.

Theorem 3.4. For every $A \in$ GIVIFS $_{B}$ and $\alpha \in[0,1]$, it holds that

$$
\begin{array}{cl}
\text { i. } & \mathrm{d}_{\alpha}(A) \in \mathrm{GIVIFS}_{\mathrm{B}}, \\
\text { ii. } & \mathrm{d}_{0}(\mathrm{~A})=\overline{\nabla A}, \\
\text { iii. } & \mathrm{d}_{1}(\mathrm{~A})=\overline{\overline{\mathrm{A}}}, \\
\text { iv. } & \mathrm{d}_{\alpha}(\bar{A})=\overline{\mathrm{d}_{1-\alpha}(\mathrm{A})}=\mathrm{D}_{\alpha}(\mathrm{A}), \\
\text { v. } & \mathrm{d}_{\alpha}\left(\mathrm{d}_{\alpha}(\mathrm{A})\right)=\mathrm{D}_{1-\alpha}(\mathrm{A}) .
\end{array}
$$

Proof. The proof of (i) is obvious.

(ii) Follows since

$$
\begin{aligned}
\mathrm{M}_{\mathrm{d}_{0}}(\mathrm{~A}) & =\left[\mathrm{N}_{\mathrm{AL}}(\mathrm{x}),\left(\mathrm{N}_{\mathrm{AU}}(\mathrm{x})^{\delta}+0 \pi_{\mathrm{AL}}(\mathrm{x})^{\delta}\right)^{\frac{1}{\delta}}\right], \\
& =\left[\mathrm{N}_{\mathrm{AL}}(\mathrm{x}), \mathrm{N}_{\mathrm{AU}}(\mathrm{x})\right], \\
\mathrm{N}_{\mathrm{d}_{0}}(\mathrm{~A}) & =\left[\mathrm{M}_{\mathrm{AL}}(\mathrm{x}),\left(\mathrm{M}_{\mathrm{AU}}(\mathrm{x})^{\delta}+(1-0) \pi_{\mathrm{AL}}(\mathrm{x})^{\delta}\right)^{\frac{1}{\delta}}\right], \\
& =\left[\mathrm{M}_{\mathrm{AL}}(\mathrm{x}),\left(\mathrm{M}_{\mathrm{AU}}(\mathrm{x})^{\delta}+\pi_{\mathrm{AL}}(\mathrm{x})^{\delta}\right)^{\frac{1}{\delta}}\right], \\
& =\left[\mathrm{M}_{\mathrm{AL}}(\mathrm{x}),\left(1-\mathrm{N}_{\mathrm{AU}}(\mathrm{x})^{\delta}\right)^{\frac{1}{\delta}}\right] .
\end{aligned}
$$

then

$$
\mathrm{d}_{0}(\mathrm{~A})=\left\{\left\langle\mathrm{x},\left[\mathrm{N}_{\mathrm{AL}}(\mathrm{x}), \mathrm{N}_{\mathrm{AU}}(\mathrm{x})\right],\left[\mathrm{M}_{\mathrm{AL}}(\mathrm{x}),\left(1-\mathrm{N}_{\mathrm{AU}}(\mathrm{x})^{\delta}\right)^{\frac{1}{\delta}}\right]\right\rangle: \mathrm{x} \in \mathrm{X}\right\}=\overline{\nabla \mathrm{A}}
$$

(iii) Follows since

$$
\begin{aligned}
& \mathrm{d}_{1}(\mathrm{~A})=\left\{\left\langle\mathrm{x}, \mathrm{M}_{\mathrm{d}_{1}}(\mathrm{~A}), \mathrm{N}_{\mathrm{d}_{1}}(\mathrm{~A})\right\rangle: \mathrm{x} \in \mathrm{X}\right\}, \\
& \begin{aligned}
\mathrm{N}_{\mathrm{d}_{1}}(\mathrm{~A}) & =\left[\mathrm{M}_{\mathrm{AL}}(\mathrm{x}),\left(\mathrm{M}_{\mathrm{AU}}(\mathrm{x})^{\delta}+(1-1) \pi_{\mathrm{AL}}(\mathrm{x})^{\delta}\right)^{\frac{1}{\delta}}\right], \\
& =\left[\mathrm{M}_{\mathrm{AL}}(\mathrm{x}), \mathrm{M}_{\mathrm{AU}}(\mathrm{x})\right], \\
\mathrm{M}_{\mathrm{d}_{1}}(\mathrm{~A}) & =\left[\mathrm{N}_{\mathrm{AL}}(\mathrm{x}),\left(\mathrm{N}_{\mathrm{AU}}(\mathrm{x})^{\delta}+\pi_{\mathrm{AL}}(\mathrm{x})^{\delta}\right)^{\frac{1}{\delta}}\right], \\
& =\left[\mathrm{N}_{\mathrm{AL}}(\mathrm{x}),\left(1-\mathrm{M}_{\mathrm{AU}}(\mathrm{x})^{\delta}\right)^{\frac{1}{\delta}}\right], \\
\mathrm{d}_{1}(\mathrm{~A})= & \left\{\left\langle\mathrm{x},\left[\mathrm{N}_{\mathrm{AL}}(\mathrm{x}),\left(1-\mathrm{M}_{\mathrm{AU}}(\mathrm{x})^{\delta}\right)^{\frac{1}{\delta}}\right],\left[\mathrm{M}_{\mathrm{AL}}(\mathrm{x}), \mathrm{M}_{\mathrm{AU}}(\mathrm{x})\right]\right\rangle: \mathrm{x} \in \mathrm{X}\right\}=\overline{\square A} .
\end{aligned}
\end{aligned}
$$

This completes the proof.

(iv) The proof of this paper is analogous to the proof of part (vi) in Theorem 3.2. The proof of part (v) is straightforward. 
Definition 3.5. Let $A \in \operatorname{GIVIFS}_{\mathrm{B}}$ and $\alpha, \beta \in[0,1]$, where $0 \leq \alpha+\beta \leq 1$, we define the operator of $\mathrm{f}_{\alpha, \beta}(A, \delta)$ (in summary, we will show as $\mathrm{f}_{\alpha, \beta}(A)$ ) as follows

$$
\begin{aligned}
& \mathrm{f}_{\alpha, \beta}(\mathrm{A})=\left\{\left\langle\mathrm{x}, \mathrm{M}_{\mathrm{f}_{\alpha}}(\mathrm{A}), \mathrm{N}_{\mathrm{f}_{\beta}}(\mathrm{A})\right\rangle: \mathrm{x} \in \mathrm{X}\right\} \\
& \mathrm{M}_{\mathrm{f}_{\alpha}}(\mathrm{A})=\left[\mathrm{N}_{\mathrm{AL}}(\mathrm{x}),\left(\mathrm{N}_{\mathrm{AU}}(\mathrm{x})^{\delta}+\alpha \pi_{\mathrm{AL}}(\mathrm{x})^{\delta}\right)^{\frac{1}{\delta}}\right], \mathrm{N}_{\mathrm{f}_{\beta}}(\mathrm{A})=\left[\mathrm{M}_{\mathrm{AL}}(\mathrm{x}),\left(\mathrm{M}_{\mathrm{AU}}(\mathrm{x})^{\delta}+\beta \pi_{\mathrm{AL}}(\mathrm{x})^{\delta}\right)^{\frac{1}{\delta}}\right] .
\end{aligned}
$$

Theorem 3.5. For every GIVIFS $S_{B} \in A$ and $\alpha, \beta, \gamma \in[0,1]$, where $0 \leq \alpha+\beta \leq 1$, it holds that

$$
\begin{array}{rll}
\text { i. } & \mathrm{f}_{\alpha, \beta}(A) \in \operatorname{GIVIFS}_{B}, \\
\text { ii. } & 0 \leq \gamma \leq \alpha \Rightarrow \mathrm{f}_{\gamma, \beta}(A) \subset \mathrm{f}_{\alpha, \beta}(A), \quad 0 \leq \gamma+\beta \leq 1, \\
\text { iii. } & 0 \leq \gamma \leq \beta \Rightarrow \mathrm{f}_{\alpha, \beta}(A) \subset \mathrm{f}_{\alpha, \gamma}(A), \quad 0 \leq \alpha+\gamma \leq 1, \\
\text { iv. } & \mathrm{f}_{\alpha, 1-\alpha}(A)=\mathrm{d}_{\alpha}(A), \\
\text { v. } & \mathrm{f}_{0,1}(A)=\overline{\Delta A} \\
\text { vi. } & \mathrm{f}_{1,0}(A)=\overline{\square A} \\
\text { vii. } & \mathrm{f}_{\alpha, \beta}(\bar{A})=\mathrm{f}_{\beta, \alpha}(A), \\
\text { viii. } & \mathrm{f}_{0,0}(A)=\bar{A}, \\
\text { ix. } & \mathrm{d}_{\alpha}(A) \subset \mathrm{f}_{\alpha, \beta}(A) .
\end{array}
$$

Proof. (i) Follows since

$$
\begin{aligned}
\mathrm{M}_{\mathrm{f}_{\alpha, \beta}(\mathrm{A}) \mathrm{U}}(\mathrm{x})^{\delta}+\mathrm{N}_{\mathrm{f}_{\alpha, \beta}(\mathrm{A}) \mathrm{U}}(\mathrm{x})^{\delta} & \\
& =\left[\left(\mathrm{N}_{\mathrm{AU}}(\mathrm{x})^{\delta}+\alpha \pi_{\mathrm{AL}}(\mathrm{x})^{\delta}\right)^{\frac{1}{\delta}}\right]^{\delta}+\left[\left(\mathrm{M}_{\mathrm{AU}}(\mathrm{x})^{\delta}+\beta \pi_{\mathrm{AL}}(\mathrm{x})^{\delta}\right)^{\frac{1}{\delta}}\right]^{\delta} \\
& =\mathrm{N}_{\mathrm{AU}}(\mathrm{x})^{\delta}+\alpha \pi_{\mathrm{AL}}(\mathrm{x})^{\delta}+\mathrm{M}_{\mathrm{AU}}(\mathrm{x})^{\delta}+\beta \pi_{\mathrm{AL}}(\mathrm{x})^{\delta} \\
& =\mathrm{M}_{\mathrm{AU}}(\mathrm{x})^{\delta}+\mathrm{N}_{\mathrm{AU}}(\mathrm{x})^{\delta}+(\alpha+\beta) \pi_{\mathrm{AL}}(\mathrm{x})^{\delta} \\
& \leq \mathrm{M}_{\mathrm{AU}}(\mathrm{x})^{\delta}+\mathrm{N}_{\mathrm{AU}}(\mathrm{x})^{\delta}+\pi_{\mathrm{AL}}(\mathrm{x})^{\delta}=1 .
\end{aligned}
$$

Proofs of (ii) and (iii) are obvious.

(iv) Since

$\mathrm{M}_{\mathrm{f}_{\alpha}}(\mathrm{A})=\left[\mathrm{N}_{\mathrm{AL}}(\mathrm{x}),\left(\mathrm{N}_{\mathrm{AU}}(\mathrm{x})^{\delta}+\alpha \pi_{\mathrm{AL}}(\mathrm{x})^{\delta}\right)^{\frac{1}{\delta}}\right], \mathrm{N}_{\mathrm{f}_{1-\alpha}}(\mathrm{A})=\left[\mathrm{M}_{\mathrm{AL}}(\mathrm{x}),\left(\mathrm{M}_{\mathrm{AU}}(\mathrm{x})^{\delta}+(1-\alpha) \pi_{\mathrm{AL}}(\mathrm{x})^{\delta}\right)^{\frac{1}{\delta}}\right]$

then

$\mathrm{f}_{\alpha, 1-\alpha}(\mathrm{A})=\left\{\left\langle\mathrm{x}, \mathrm{M}_{\mathrm{f}_{\alpha}}(\mathrm{A}), \mathrm{N}_{\mathrm{f}_{1-\alpha}}(\mathrm{A})\right\rangle: \mathrm{x} \in \mathrm{X}\right\}=\mathrm{d}_{\alpha}(\mathrm{A})$.

(v) Since $\mathrm{f}_{0,1}(\mathrm{~A})=\mathrm{d}_{0}(\mathrm{~A})$ by using Theorem 3.4 it follows that $\mathrm{f}_{0,1}(\mathrm{~A})=\overline{\nabla \mathrm{A}}$.

(vi) Since $f_{1,0}(A)=d_{1}(A)$ by using Theorem 3.4 it follows that $f_{1,0}(A)=\overline{\square A}$.

(vii) Since

$$
\begin{aligned}
& \mathrm{f}_{\beta, \alpha}(A)=\left\{\left\langle\mathrm{x}, \mathrm{M}_{\mathrm{f}_{\beta}}(\mathrm{A}), \mathrm{N}_{\mathrm{f}_{\alpha}}(\mathrm{A})\right\rangle: \mathrm{x} \in \mathrm{X}\right\}, \\
& \mathrm{M}_{\mathrm{f}_{\beta}}(\mathrm{A})=\left[\mathrm{N}_{\mathrm{AL}}(\mathrm{x}),\left(\mathrm{N}_{\mathrm{AU}}(\mathrm{x})^{\delta}+\beta \pi_{\mathrm{AL}}(\mathrm{x})^{\delta}\right)^{\frac{1}{\delta}}\right], \mathrm{N}_{\mathrm{f}_{\alpha}}(\mathrm{A})=\left[\mathrm{M}_{\mathrm{AL}}(\mathrm{x}),\left(\mathrm{M}_{\mathrm{AU}}(\mathrm{x})^{\delta}+\alpha \pi_{\mathrm{AL}}(\mathrm{x})^{\delta}\right)^{\frac{1}{\delta}}\right] \\
& \text { and } \\
& \mathrm{f}_{\alpha, \beta}(\overline{\mathrm{A}})=\left\{\left\langle\mathrm{x}, \mathrm{M}_{\mathrm{f}_{\alpha}}(\overline{\mathrm{A}}), \mathrm{N}_{\mathrm{f}_{\beta}}(\overline{\mathrm{A}})\right\rangle: \mathrm{x} \in \mathrm{X}\right\},
\end{aligned}
$$


$\mathrm{M}_{\mathrm{f}_{\alpha}}(\overline{\mathrm{A}})=\left[\mathrm{M}_{\mathrm{AL}}(\mathrm{x}),\left(\mathrm{M}_{\mathrm{AU}}(\mathrm{x})^{\delta}+\alpha \pi_{\mathrm{AL}}(\mathrm{x})^{\delta}\right)^{\frac{1}{\delta}}\right], \mathrm{N}_{\mathrm{f}_{\beta}}(\overline{\mathrm{A}})=\left[\mathrm{N}_{\mathrm{AL}}(\mathrm{x}),\left(\mathrm{N}_{\mathrm{AU}}(\mathrm{x})^{\delta}+\beta \pi_{\mathrm{AL}}(\mathrm{x})^{\delta}\right)^{\frac{1}{\delta}}\right]$

hence

$$
\overline{\mathrm{f}_{\alpha, \beta}(\overline{\mathrm{A}})}=\left\{\left\langle\mathrm{x}, \mathrm{N}_{\mathrm{f}_{\beta}}(\overline{\mathrm{A}}), \mathrm{M}_{\mathrm{f}_{\alpha}}(\overline{\mathrm{A}})\right\rangle: \mathrm{x} \in \mathrm{X}\right\} .
$$

Finally, we have $\overline{f_{\alpha, \beta}(\bar{A})}=f_{\beta, \alpha}(A)$. The proof of part (viii) is straightforward.

(ix) It follows from the fact that $\beta \leq 1-\alpha$.

Theorem 3.6. For every $A \in$ GIVIFS $_{B}$ and $\alpha, \beta \in[0,1]$, where $0 \leq \alpha+\beta \leq 1$, it holds that

$$
\begin{array}{cl}
\text { i. } & \mathrm{D}_{\alpha}(\square \mathrm{A})=\mathrm{F}_{\alpha, \beta}(\square \mathrm{A})=\square \mathrm{A}, \\
\text { ii. } & \mathrm{D}_{\alpha}(\diamond \mathrm{A})=\mathrm{F}_{\alpha, \beta}(\diamond \mathrm{A})=\Delta \mathrm{A}, \\
\text { iii. } & \mathrm{d}_{\alpha}(\square \mathrm{A})=\mathrm{f}_{\alpha, \beta}(\square \mathrm{A})=\overline{\square A}, \\
\text { iv. } & \mathrm{d}_{\alpha}(\diamond \mathrm{A})=\mathrm{f}_{\alpha, \beta}(\diamond \mathrm{A})=\overline{\nabla A} .
\end{array}
$$

Proof. Proof of the theorem is obtained directly from the definitions.

Theorem 3.7. For every A $\in$ GIVIFS $_{B}$ and $\alpha_{1}, \alpha_{2}, \eta, \gamma \in[0,1]$, where $0 \leq \eta+\gamma \leq 1$, it holds that
i. $\quad d_{\alpha_{1}}\left(d_{\alpha_{2}}(A)\right)=D_{1-\alpha_{2}}(A)$,
ii. $\mathrm{D}_{\alpha_{1}}\left(\mathrm{D}_{\alpha_{2}}(\mathrm{~A})\right)=\mathrm{D}_{\alpha_{2}}(\mathrm{~A})$,
iii. $d_{\alpha_{1}}\left(D_{\alpha_{2}}(A)\right)=d_{1-\alpha_{2}}(A)$,
iv. $\quad D_{\alpha_{1}}\left(d_{\alpha_{2}}(A)\right)=d_{\alpha_{2}}(A)$,
v. $\quad F_{\eta, \gamma}\left(D_{\alpha_{2}}(A)\right)=D_{\alpha_{2}}(A)$,
vi. $\quad F_{\eta, \gamma}\left(d_{\alpha_{2}}(A)\right)=d_{\alpha_{2}}(A)$,
vii. $f_{\eta, \gamma}\left(D_{\alpha_{2}}(A)\right)=d_{1-\alpha_{2}}(A)$,
viii. $\mathrm{f}_{\eta, \gamma}\left(\mathrm{d}_{\alpha_{2}}(\mathrm{~A})\right)=\mathrm{D}_{1-\alpha_{2}}(\mathrm{~A})$.

Proof. Proof of the theorem is obtained directly from the definitions.

It can be easily shown that $F_{\eta, \gamma}\left(D_{\alpha}(A)\right)=\overline{F_{\eta, \gamma}\left(d_{1-\alpha}(A)\right)}, f_{\eta, \gamma}\left(d_{1-\alpha}(A)\right)=F_{\eta, \gamma}\left(D_{\alpha}(A)\right)$, and
$d_{\alpha_{1}}\left(D_{1-\alpha_{2}}(A)\right)=D_{\alpha_{1}}\left(d_{\alpha_{2}}(A)\right)$.

Definition 3.6. Let $A \in \operatorname{GIVIFS}_{B}$ and $\alpha, \beta \in[0,1]$, we define the operator of $j_{\alpha, \beta}(A, \delta)$ (in summary, we will show as $\left.\mathrm{j}_{\alpha, \beta}(A)\right)$ as follows

$$
\begin{aligned}
& \mathrm{j}_{\alpha, \beta}(\mathrm{A})=\left\{\left\langle\mathrm{x}, \mathrm{M}_{\mathrm{j}_{\alpha, \beta}}(\mathrm{A}), \mathrm{N}_{\mathrm{j}_{\alpha, \beta}}(\mathrm{A})\right\rangle: \mathrm{x} \in \mathrm{X}\right\} \\
& \mathrm{M}_{\mathrm{j}_{\alpha, \beta}}(\mathrm{A})=\left[\mathrm{N}_{\mathrm{AL}}(\mathrm{x}),\left(\mathrm{N}_{\mathrm{AU}}(\mathrm{x})^{\delta}+\alpha \pi_{\mathrm{AL}}(\mathrm{x})^{\delta}\right)^{\frac{1}{\delta}}\right], \mathrm{N}_{\mathrm{j}_{\alpha, \beta}}(\mathrm{A})=\left[\beta^{\frac{1}{\delta}} \mathrm{M}_{\mathrm{AL}}(\mathrm{x}), \beta^{\frac{1}{\delta}} \mathrm{M}_{\mathrm{AU}}(\mathrm{x})\right]
\end{aligned}
$$

Theorem 3.8. For every $A \in \operatorname{GIVIFS}_{B}$ and $\alpha, \beta, \gamma \in[0,1]$, it holds that

$$
\text { i. } \quad \mathrm{j}_{\alpha, \beta}(\mathrm{A}) \in \mathrm{GIVIFS}_{\mathrm{B}}
$$




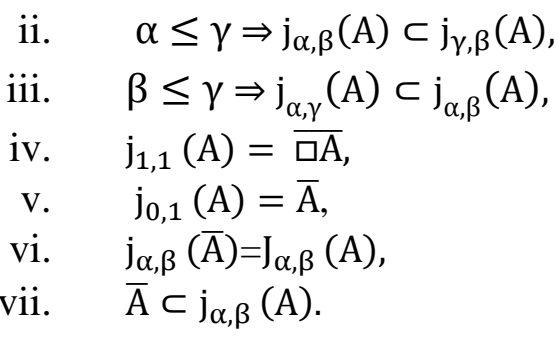

Proof. (i) Note that

$$
\begin{aligned}
\mathrm{M}_{\mathrm{j}_{\alpha, \beta}(\mathrm{A}) \mathrm{U}}(\mathrm{x})^{\delta}+\mathrm{N}_{\mathrm{j}_{\alpha, \beta}(\mathrm{A}) \mathrm{U}}(\mathrm{x})^{\delta} & \left.=\left(\mathrm{N}_{\mathrm{AU}}(\mathrm{x})^{\delta}+\alpha \pi_{\mathrm{AL}}(\mathrm{x})^{\delta}\right)^{\frac{1}{\delta}}\right)^{\delta}+\left(\beta^{\frac{1}{\delta}} \mathrm{M}_{\mathrm{AU}}(\mathrm{x})\right)^{\delta}, \\
& =\left(\mathrm{N}_{\mathrm{AU}}(\mathrm{x})^{\delta}+\alpha \pi_{\mathrm{AL}}(\mathrm{x})^{\delta}\right)+\beta \mathrm{M}_{\mathrm{AU}}(\mathrm{x})^{\delta} \\
& \leq \mathrm{N}_{\mathrm{AU}}(\mathrm{x})^{\delta}+\pi_{\mathrm{AL}}(\mathrm{x})^{\delta}+\mathrm{M}_{\mathrm{AU}}(\mathrm{x})^{\delta}=1 .
\end{aligned}
$$

Finally, it can be concluded that $j_{\alpha, \beta}(A) \in \operatorname{GIVIFS}_{B}$.

(ii) Since $\alpha \leq \gamma$ then it is clear that

$\left[\mathrm{N}_{\mathrm{AL}}(\mathrm{x}),\left(\mathrm{N}_{\mathrm{AU}}(\mathrm{x})^{\delta}+\alpha \pi_{\mathrm{AL}}(\mathrm{x})^{\delta}\right)^{\frac{1}{\delta}}\right] \leq\left[\mathrm{N}_{\mathrm{AL}}(\mathrm{x}),\left(\mathrm{N}_{\mathrm{AU}}(\mathrm{x})^{\delta}+\gamma \pi_{\mathrm{AL}}(\mathrm{x})^{\delta}\right)^{\frac{1}{\delta}}\right]$

Finally we have $j_{\alpha, \beta}(A) \subset j_{\gamma, \beta}(A)$.

The proof of (iii) is similar to that of (ii). Proofs of (iv), (v), (vi) and (vii) are obvious.

Definition 3.7. Let $A \in$ GIVIFS $_{B}$ and $\alpha, \beta \in[0,1]$, we define the operator of $H_{\alpha, \beta}(A, \delta)$ (in summary, we will show as $\left.\mathrm{H}_{\alpha, \beta}(\mathrm{A})\right)$ as follows

$$
\begin{aligned}
\mathrm{H}_{\alpha, \beta}(\mathrm{A}) & =\left\{\left\langle\mathrm{x}, \mathrm{M}_{\mathrm{H}_{\alpha, \beta}}(\mathrm{A}), \mathrm{N}_{\mathrm{H}_{\alpha, \beta}}(\mathrm{A})\right\rangle: \mathrm{x} \in \mathrm{X}\right\}, \\
\mathrm{M}_{\mathrm{H}_{\alpha, \beta}}(\mathrm{A}) & =\left[\alpha^{\frac{1}{\delta}} \mathrm{M}_{\mathrm{AL}}(\mathrm{x}), \alpha^{\frac{1}{\delta}} \mathrm{M}_{\mathrm{AU}}(\mathrm{x})\right], \mathrm{N}_{\mathrm{H}_{\alpha, \beta}}(\mathrm{A})=\left[\left(\mathrm{N}_{\mathrm{AL}}(\mathrm{x}),\left(\mathrm{N}_{\mathrm{AU}}(\mathrm{x})^{\delta}+\beta \pi_{\mathrm{AL}}(\mathrm{x})^{\delta}\right)^{\frac{1}{\delta}}\right] .\right.
\end{aligned}
$$

Theorem 3.9. For every $A \in$ GIVIFS $_{B}$ and $\alpha, \beta, \gamma \in[0,1]$ it holds that

i. $\mathrm{H}_{\alpha, \beta}(\mathrm{A}) \in \mathrm{GIVIFS}_{\mathrm{B}}$,

ii. $\quad \alpha \leq \gamma \Rightarrow \mathrm{H}_{\gamma, \beta}(\mathrm{A}) \subset \mathrm{H}_{\alpha, \beta}(\mathrm{A})$,

iii. $\quad \beta \leq \gamma \Rightarrow \mathrm{H}_{\alpha, \gamma}(\mathrm{A}) \subset \mathrm{H}_{\alpha, \beta}(\mathrm{A})$,

iv. $\mathrm{H}_{1,0}(\mathrm{~A})=\mathrm{A}$,

v. $\mathrm{H}_{1,1}(\mathrm{~A})=\square \mathrm{A}$,

vi. $\mathrm{H}_{\alpha, \beta}(\mathrm{A}) \subset \mathrm{A}$.

Proof. (i) Follows since

$$
\begin{aligned}
\mathrm{M}_{\mathrm{H}_{\alpha, \beta}(\mathrm{A}) \mathrm{U}}(\mathrm{x})^{\delta}+\mathrm{N}_{\mathrm{H}_{\alpha, \beta}(\mathrm{A}) \mathrm{U}}(\mathrm{x})^{\delta} & \left.=\left(\alpha^{\frac{1}{\delta}} \mathrm{M}_{\mathrm{AU}}(\mathrm{x})\right)^{\delta}+\left(\mathrm{N}_{\mathrm{AU}}(\mathrm{x})^{\delta}+\beta \pi_{\mathrm{AL}}(\mathrm{x})^{\delta}\right)^{\frac{1}{\delta}}\right)^{\delta}, \\
& =\alpha \mathrm{M}_{\mathrm{AU}}(\mathrm{x})^{\delta}+\left(\mathrm{N}_{\mathrm{AU}}(\mathrm{x})^{\delta}+\beta \pi_{\mathrm{AL}}(\mathrm{x})^{\delta}\right) \\
& \leq \mathrm{M}_{\mathrm{AU}}(\mathrm{x})^{\delta}+\mathrm{N}_{\mathrm{AU}}(\mathrm{x})^{\delta}+\pi_{\mathrm{AL}}(\mathrm{x})^{\delta}=1 .
\end{aligned}
$$

Proofs of (ii), (iii), (iv), (v) and (vi) are obvious. 
Definition 3.8. Let $A \in \operatorname{GIVIFS}_{B}$ and $\alpha, \beta \in[0,1]$, we define the operator of $h_{\alpha, \beta}(A, \delta)$ (in summary, we will show as $\left.\mathrm{h}_{\alpha, \beta}(\mathrm{A})\right)$ as follows

$$
\begin{aligned}
& \mathrm{h}_{\alpha, \beta}(\mathrm{A})=\left\{\left\langle\mathrm{x}, \mathrm{M}_{\mathrm{h}_{\alpha, \beta}}(\mathrm{A}), \mathrm{N}_{\mathrm{h}_{\alpha, \beta}}(\mathrm{A})\right\rangle: \mathrm{x} \in \mathrm{X}\right\}, \\
& \mathrm{M}_{\mathrm{h}_{\alpha, \beta}}(\mathrm{A})=\left[\alpha^{\frac{1}{\delta}} \mathrm{N}_{\mathrm{AL}}(\mathrm{x}), \alpha^{\frac{1}{\delta}} \mathrm{N}_{\mathrm{AU}}(\mathrm{x})\right], \mathrm{N}_{\mathrm{h}_{\alpha, \beta}}(\mathrm{A})=\left[\left(\mathrm{M}_{\mathrm{AL}}(\mathrm{x}),\left(\mathrm{M}_{\mathrm{AU}}(\mathrm{x})^{\delta}+\beta \pi_{\mathrm{AL}}(\mathrm{x})^{\delta}\right)^{\frac{1}{\delta}}\right] .\right.
\end{aligned}
$$

Theorem 3.10. For every $A \in \operatorname{GIVIFS}_{B}$ and $\alpha, \beta, \gamma \in[0,1]$, it holds that

$$
\begin{array}{cl}
\text { i. } & h_{\alpha, \beta}(A) \in \operatorname{GIVIFS}_{B}, \\
\text { ii. } & \alpha \leq \gamma \Rightarrow h_{\gamma, \beta}(A) \subset h_{\alpha, \beta}(A), \\
\text { iii. } & \beta \leq \gamma \Rightarrow h_{\alpha, \gamma}(A) \subset h_{\alpha, \beta}(A), \\
\text { iv. } & h_{\alpha, \beta}(\bar{A})=H_{\alpha, \beta}(A) \\
\text { v. } & h_{1,0}(A)=\bar{A} \\
\text { vi. } & h_{1,1}(A)=\bar{\nabla} \\
\text { vii. } & h_{\alpha, \beta}(A) \subset \bar{A} .
\end{array}
$$

Proof. (i) Follows since

$$
\begin{aligned}
\mathrm{M}_{\mathrm{h}_{\alpha, \beta}(\mathrm{A}) \mathrm{U}}(\mathrm{x})^{\delta}+\mathrm{N}_{\mathrm{h}_{\alpha, \beta}(\mathrm{A}) \mathrm{U}}(\mathrm{x})^{\delta} & =\left(\alpha^{\frac{1}{\delta}} \mathrm{N}_{\mathrm{AU}}(\mathrm{x})\right)^{\delta}+\left(\left(\mathrm{M}_{\mathrm{AU}}(\mathrm{x})^{\delta}+\beta \pi_{\mathrm{AL}}(\mathrm{x})^{\delta}\right)^{\frac{1}{\delta}}\right)^{\delta}, \\
& =\alpha \mathrm{N}_{\mathrm{AU}}(\mathrm{x})^{\delta}+\left(\mathrm{M}_{\mathrm{AU}}(\mathrm{x})^{\delta}+\beta \pi_{\mathrm{AL}}(\mathrm{x})^{\delta}\right), \\
& \leq \mathrm{N}_{\mathrm{AU}}(\mathrm{x})^{\delta}+\mathrm{M}_{\mathrm{AU}}(\mathrm{x})^{\delta}+\pi_{\mathrm{AL}}(\mathrm{x})^{\delta}=1 .
\end{aligned}
$$

Proofs of (ii), (iii), (iv), (v), (vi) and (vii) are obvious.

Theorem 3.11. For every $A \in \operatorname{GIVIFS}_{B}$ and $\alpha, \beta, \gamma \in[0,1]$, it holds that

$$
\begin{array}{cl}
\text { i. } & \mathrm{H}_{\alpha, \beta}\left(\mathrm{D}_{\alpha}(\mathrm{A})\right) \subset \mathrm{D}_{\alpha}(\mathrm{A}) \subset \mathrm{J}_{\alpha, \beta}\left(\mathrm{D}_{\alpha}(\mathrm{A})\right), \\
\text { ii. } & \mathrm{H}\left(\mathrm{d}_{\alpha}(A)\right) \subset \mathrm{d}_{\alpha}(\mathrm{A}) \subset \mathrm{J}_{\alpha, \beta}\left(\mathrm{d}_{\alpha}(A)\right), \\
\text { iii. } & \mathrm{h}\left(\mathrm{d}_{\alpha}(\mathrm{A})\right) \subset \mathrm{D}_{1-\alpha}(A) \subset \mathrm{j}_{\alpha, \beta}\left(\mathrm{d}_{\alpha}(A)\right), \\
\text { iv. } & \mathrm{h}\left(\mathrm{D}_{\alpha}(A)\right) \subset \mathrm{d}_{1-\alpha}(A) \subset \mathrm{j}_{\alpha, \beta}\left(\mathrm{D}_{\alpha}(A)\right) .
\end{array}
$$

Proof. Proof of the theorem is obtained directly from the definitions.

Theorem 3.12. For every $A \in \operatorname{GIVIFS}_{\mathrm{B}}$ and $\alpha, \beta, \in[0,1]$, where $0 \leq \alpha+\beta \leq 1, \delta_{1} \leq \delta_{2}$, it holds that

$$
\begin{array}{cl}
\text { i. } & \mathrm{D}_{\alpha}\left(\mathrm{A}, \delta_{2}\right) \subset_{\square} \mathrm{D}_{\alpha}\left(\mathrm{A}, \delta_{1}\right) \text { and } \mathrm{D}_{\alpha}\left(\mathrm{A}, \delta_{1}\right) \subset_{\diamond} \mathrm{D}_{\alpha}\left(\mathrm{A}, \delta_{2}\right), \\
\text { ii. } & \mathrm{F}_{\alpha, \beta}\left(\mathrm{A}, \delta_{2}\right) \subset_{\square} \mathrm{F}_{\alpha, \beta}\left(\mathrm{A}, \delta_{1}\right) \text { and } \mathrm{F}_{\alpha, \beta}\left(\mathrm{A}, \delta_{1}\right) \subset_{\diamond} \mathrm{F}_{\alpha, \beta}\left(\mathrm{A}, \delta_{2}\right), \\
\text { iii. } & \mathrm{J}_{\alpha, \beta}\left(\mathrm{A}, \delta_{2}\right) \subset \mathrm{J}_{\alpha, \beta}\left(\mathrm{A}, \delta_{1}\right), \\
\text { iv. } & \mathrm{d}_{\alpha}\left(\mathrm{A}, \delta_{2}\right) \subset_{\square} \mathrm{d}_{\alpha}\left(\mathrm{A}, \delta_{1}\right) \text { and } \mathrm{d}_{\alpha}\left(\mathrm{A}, \delta_{1}\right) \subset_{\diamond} \mathrm{d}_{\alpha}\left(\mathrm{A}, \delta_{2}\right), \\
\text { v. } & \mathrm{f}_{\alpha, \beta}\left(\mathrm{A}, \delta_{2}\right) \subset_{\square} \mathrm{f}_{\alpha, \beta}\left(\mathrm{A}, \delta_{1}\right) \text { and } \mathrm{f}_{\alpha, \beta}\left(\mathrm{A}, \delta_{1}\right) \subset_{\diamond} \mathrm{f}_{\alpha, \beta}\left(\mathrm{A}, \delta_{2}\right), \\
\text { vi. } & \mathrm{j}_{\alpha, \beta}\left(\mathrm{A}, \delta_{2}\right) \subset \mathrm{j}_{\alpha, \beta}\left(\mathrm{A}, \delta_{1}\right), \\
\text { vii. } & \mathrm{H}_{\alpha, \beta}\left(\mathrm{A}, \delta_{1}\right) \subset \mathrm{H}_{\alpha, \beta}\left(\mathrm{A}, \delta_{2}\right), \\
\text { viii. } & \mathrm{h}_{\alpha, \beta}\left(\mathrm{A}, \delta_{1}\right) \subset \mathrm{h}_{\alpha, \beta}\left(\mathrm{A}, \delta_{2}\right) .
\end{array}
$$

Proof. It follows from the fact that $g_{1}(\delta)=\left(a^{\delta}+\alpha b^{\delta}\right)^{\frac{1}{\delta}}$ is decreasing and $g_{2}(\delta)=a^{\frac{1}{\delta}}$ is increasing.

Corollary 3.1. For every $A \in \operatorname{GIVIFS}_{\mathrm{B}}$, where $\mathrm{M}_{\mathrm{AU}}(\mathrm{x})^{\delta}+\mathrm{N}_{\mathrm{AU}}(\mathrm{x})^{\delta}=1$, it holds it 
i. $\quad D_{\alpha}(A)=F_{\alpha, \beta}(A)=A$,

ii. $\quad \mathrm{d}_{\alpha}(\mathrm{A})=\mathrm{f}_{\alpha, \beta}(\mathrm{A})=\overline{\mathrm{A}}$.

Corollary 3.2. For every $A \in$ GIVIFS $_{B}$ and $\alpha_{i}, \beta_{i} \in[0,1]$, where $\alpha_{1} \leq \alpha_{2}, \beta_{2} \leq \beta_{1}$ and $0 \leq \alpha_{i}+\beta_{i} \leq 1$ $, \mathrm{i}=1,2$, it holds that
i. $\quad \mathrm{D}_{\alpha_{1}}(\mathrm{~A}) \subset \mathrm{D}_{\alpha_{2}}(\mathrm{~A})$,
ii. $\quad \mathrm{d}_{\alpha_{1}}(\mathrm{~A}) \subset \mathrm{d}_{\alpha_{2}}(\mathrm{~A})$,
iii. $\quad \mathrm{F}_{\alpha_{1}, \beta_{1}}(\mathrm{~A}) \subset \mathrm{F}_{\alpha_{2}, \beta_{2}}(\mathrm{~A})$,
iv. $\quad J_{\alpha_{1}, \beta_{1}}(A) \subset J_{\alpha_{2}, \beta_{2}}(A)$,
v. $\quad \mathrm{j}_{\alpha_{1}, \beta_{1}}(A) \subset \mathrm{j}_{\alpha_{2}, \beta_{2}}(A)$,
vi. $\quad f_{\alpha_{1}, \beta_{1}}(A) \subset f_{\alpha_{2}, \beta_{2}}(A)$,
vii. $\quad H_{\alpha_{1}, \beta_{1}}(A) \subset \mathrm{H}_{\alpha_{2}, \beta_{2}}(A)$,
viii. $\quad h_{\alpha_{1}, \beta_{1}}(A) \subset h_{\alpha_{2}, \beta_{2}}(A)$.

Corollary 3.3. For every $A \in \operatorname{GIVIFS}_{\mathrm{B}}$ and $\alpha, \beta \in[0,1]$, it holds that
i. $\quad \mathrm{d}_{\alpha}(A) \subset \mathrm{f}_{\alpha, \beta}(A) \subset \mathrm{j}_{\alpha, \beta}(A)$,
ii. $\mathrm{D}_{\alpha}(\mathrm{A}) \subset \mathrm{F}_{\alpha, \beta}(\mathrm{A}) \subset \mathrm{J}_{\alpha, \beta}(\mathrm{A})$,
iii. $\mathrm{H}_{\alpha, \beta}(A) \subset \mathrm{J}_{\alpha, \beta}(A)$,
iv. $\mathrm{h}_{\alpha, \beta}(A) \subset \mathrm{j}_{\alpha, \beta}(A)$.

Remark 3.1. According to definition, the operators of $D_{\alpha}(A)$ and $F_{\alpha, \beta}(A)$ increases the membership and non-membership degree $A$, the operators of $d_{\alpha}(A)$ and $f_{\alpha, \beta}(A)$ increases the membership and nonmembership degree $\bar{A}$, the operators of $h_{\alpha, \beta}(A)$ reduces the membership degree $\bar{A}$ and increases nonmembership degree $\bar{A}$, the operators of $H_{\alpha, \beta}(A)$ reduces the membership degree $A$ and increases nonmembership degree $A$, the operators of $j_{\alpha, \beta}(A)$ increases the membership degree $\bar{A}$ and reduces nonmembership degree $\bar{A}$, the operators of $J_{\alpha, \beta}(A)$ increases the membership degree $A$ and reduces nonmembership degree $\mathrm{A}$.

Example 3.1. Let $A=\left\{\left\langle\mathrm{x}_{1},[0.2,0.3],[0.1,0.2]\right\rangle\right\}, \delta=0.5$, then

$$
\begin{aligned}
& \square \mathrm{A}=\left\{\left\langle\mathrm{x}_{1},[0.2,0.3],[0.1,0.204555]\right\rangle\right\} \\
& \left.\left.\nabla \mathrm{A}=\left\{\left\langle\mathrm{x}_{1}, 0.2,0.305573\right], 0.10 .2\right]\right\rangle\right\} \\
& \pi_{\mathrm{AL}}\left(\mathrm{x}_{1}\right)^{0.5}=0.005064 \\
& \mathrm{~F}_{\alpha, \beta}(\mathrm{A})=\left\{\left\langle\mathrm{x}_{1},\left[0.2,(\sqrt{0.3}+0.005064 \alpha)^{2}\right],\left[0.1,(\sqrt{0.2}+0.005064 \beta)^{2}\right]\right\rangle\right\} \\
& \mathrm{f}_{\alpha, \beta}(\mathrm{A})=\left\{\left\langle\mathrm{x}_{1},\left[0.1,(\sqrt{0.2}+0.005064 \alpha)^{2}\right],\left[0.2,(\sqrt{0.3}+0.005064 \beta)^{2}\right]\right\rangle\right\} \\
& \mathrm{J}_{\alpha, \beta}(\mathrm{A})=\left\{\left\langle\mathrm{x}_{1},\left[0.2,(\sqrt{0.3}+0.005064 \alpha)^{2}\right],\left[0.1 \beta^{2}, 0.2 \beta^{2}\right]\right\rangle\right\} \\
& \mathrm{j}_{\alpha, \beta}(\mathrm{A})=\left\{\left\langle\mathrm{x}_{1},\left[0.1,(\sqrt{0.2}+0.005064 \alpha)^{2}\right],\left[0.2 \beta^{2}, 0.3 \beta^{2}\right]\right\rangle\right\} \\
& \mathrm{H}_{\alpha, \beta}(\mathrm{A})=\left\{\left\langle\mathrm{x}_{1},\left[0.2 \alpha^{2}, 0.3 \alpha^{2}\right],\left[0.1,(\sqrt{0.2}+0.005064 \beta)^{2}\right]\right\rangle\right\} \\
& \mathrm{h}_{\alpha, \beta}(\mathrm{A})=\left\{\left\langle\mathrm{x}_{1},\left[0.1 \alpha^{2}, 0.2 \alpha^{2}\right],\left[0.2,(\sqrt{0.3}+0.005064 \beta)^{2}\right]\right\rangle\right\}
\end{aligned}
$$

Example 3.2. Let $A=\left\{\left\langle\mathrm{x}_{1},[0.2,0.3],[0.1,0.2]\right\rangle\right\}, \delta=0.5$, then operators due to Baloui Jamkhaneh [41] are as follows

$\pi_{\mathrm{A}}\left(\mathrm{x}_{1}\right)=\left[0.005064^{2}, 0.236559^{2}\right], \lambda_{\mathrm{A}}=1.3032254$. 


$$
\begin{aligned}
& \mathrm{F}_{\alpha, \beta}(\mathrm{A})=\left\{\left\langle\mathrm{x}_{1},\left[(\sqrt{0.2}+0.236559 \alpha)^{2},(\sqrt{0.3}+0.005064 \alpha)^{2}\right],\left[(\sqrt{0.1}+0.236559 \beta)^{2},(\sqrt{0.2}+\right.\right.\right. \\
& \left.\left.\left.0.005064 \beta)^{2}\right]\right\rangle\right\}, 0 \leq \alpha \leq 0.4341737,0 \leq \beta \leq 0.5658262 . \\
& \mathrm{f}_{\alpha, \beta}(\mathrm{A})=\left\{\left\langle\mathrm{x}_{1},\left[(\sqrt{0.1}+0.236559 \alpha)^{2},(\sqrt{0.2}+0.005064 \alpha)^{2}\right],\left[(\sqrt{0.2}+0.236559 \beta)^{2},(\sqrt{0.3}+\right.\right.\right. \\
& \left.\left.\left.0.005064 \beta)^{2}\right]\right\rangle\right\}, 0 \leq \alpha \leq 0.5658262,0 \leq \beta \leq 0.4341737 . \\
& \mathrm{J}_{\alpha, \beta}(\mathrm{A})=\left\{\left\langle\mathrm{x}_{1},\left[(\sqrt{0.2}+0.236559 \alpha)^{2},(\sqrt{0.3}+0.005064 \alpha)^{2}\right],\left[0.1 \beta^{2}, 0.2 \beta^{2}\right]\right\rangle: \mathrm{x} \in \mathrm{X}\right\}, \\
& 0 \leq \alpha \leq 0.4341737,0 \leq \beta \leq 1 . \\
& \mathrm{j}_{\alpha, \beta}(\mathrm{A})=\left\{\left\langle\mathrm{x}_{1},\left[(\sqrt{0.1}+0.236559 \alpha)^{2},(\sqrt{0.2}+0.005064 \alpha)^{2}\right],\left[0.2 \beta^{2}, 0.3 \beta^{2}\right]\right\rangle\right\}, \\
& 0 \leq \alpha \leq 0.5658262,0 \leq \beta \leq 1 . \\
& \mathrm{H}_{\alpha, \beta}(\mathrm{A})=\left\{\left\langle\mathrm{x}_{1},\left[0.2 \alpha^{2}, 0.3 \alpha^{2}\right],\left[(\sqrt{0.1}+0.236559 \alpha)^{2},(\sqrt{0.2}+0.005064 \alpha)^{2}\right]\right\rangle\right\}, \\
& 0 \leq \alpha \leq 1,0 \leq \beta \leq 0.5658262 . \\
& \mathrm{h}_{\alpha, \beta}(\mathrm{A})=\left\{\left\langle\mathrm{x}_{1},\left[0.1 \alpha^{2}, 0.2 \alpha^{2}\right],\left[(\sqrt{0.2}+0.236559 \alpha)^{2},(\sqrt{0.3}+0.005064 \alpha)^{2},(0.36+0.55 \beta)^{\frac{1}{2}}\right]\right\rangle\right\}, \\
& 0 \leq \alpha \leq 1,0 \leq \beta \leq 0.4341737 .
\end{aligned}
$$

Remark 3.2. According to definitions and examples, only the upper bound increases for any new operator that increases the degrees. Correspondingly, the upper and lower bound increases for any operator that increases the degrees in the operators of Baloui Jamkhaneh [41]. From these comparison results, it can be seen that the proposed operators have more general parameters.

\section{CONCLUSIONS}

We have introduced modified modal types of operators over Baloui's generalized interval valued intuitionistic fuzzy sets and their relationships are proved. We show that these operators are GIVIFS $_{\mathrm{B}}$. Some proven relationships between operators are shown in Table 1. For example, cell $(1,1)$ shows that $d_{\alpha_{1}}\left(D_{\alpha_{2}}(A)\right)=d_{1-\alpha_{2}}(A)$. An open problem is: definition of level operators, negation operators and other operators over $\mathrm{GIVIFS}_{\mathrm{B}}$ and the study of their properties.

Table 1. Relation between operators

\begin{tabular}{|l|l|l|l|l|}
\hline & $D_{\alpha_{2}}(A)$ & $d_{\alpha_{2}}(A)$ & $\square A$ & $\nabla A$ \\
\hline$d_{\alpha_{1}}$ & $d_{1-\alpha_{2}}(A)$ & $D_{1-\alpha_{2}}(A)$ & $\overline{\square A}$ & $\overline{\nabla A}$ \\
\hline$D_{\alpha_{1}}$ & $D_{\alpha_{2}}(A)$ & $d_{\alpha_{2}}(A)$ & $\square A$ & $\nabla A$ \\
\hline$F_{\eta, \gamma}$ & $D_{\alpha_{2}}(A)$ & $d_{\alpha_{2}}(A)$ & $\square A$ & $\nabla A$ \\
\hline$f_{\eta, \gamma}$ & $d_{1-\alpha_{2}}(A)$ & $D_{1-\alpha_{2}}(A)$ & $\overline{\square A}$ & $\overline{\nabla A}$ \\
\hline
\end{tabular}

Table 2. Special cases of operators

\begin{tabular}{|l|l|l|l|l|}
\hline & $\mathrm{D}_{\alpha}(\mathrm{A})$ & $\mathrm{F}_{\alpha, \beta}(\mathrm{A})$ & $\mathrm{d}_{\alpha}(\mathrm{A})$ & $\mathrm{f}_{\alpha, \beta}(\mathrm{A})$ \\
\hline$\alpha=0, \beta=1$ & $\square \mathrm{A}$ & $\square \mathrm{A}$ & $\overline{\nabla \mathrm{A}}$ & $\overline{\nabla \mathrm{A}}$ \\
\hline$\alpha=1, \beta=0$ & $\diamond \mathrm{A}$ & $\diamond \mathrm{A}$ & $\overline{\square \mathrm{A}}$ & $\overline{\square \mathrm{A}}$ \\
\hline
\end{tabular}

\section{ACKNOWLEDGEMENTS}

The author wishes to thank the editor and referees, for the careful reading of the paper and for the helpful suggestions and comments.

\section{CONFLICTS OF INTEREST}

No conflict of interest was declared by the author. 


\section{REFERENCES}

[1] Atanassov, K.T., "Intuitionistic fuzzy sets”, Fuzzy Sets and Systems, 20(1): 87-96, (1986).

[2] Zadeh, L.A., "Fuzzy sets", Information and Control, 8(3): 338-356, (1965).

[3] Atanassov, K.T., Gargov G., "Interval valued intuitionistic fuzzy sets", Fuzzy Sets and Systems, 31(3): 343-349, (1989).

[4] Atanassov, K.T., "Operations over interval valued fuzzy set”, Fuzzy Sets and Systems, 64(2): 159-174, (1994).

[5] Xu, Z., "Methods for aggregating interval-valued intuitionistic fuzzy information and their application to decision making", Control and Decision, 22(2): 215, (2007).

[6] Xu, Z.S., Jian, C.H.E.N., "Approach to group decision making based on interval-valued intuitionistic judgment matrices", Systems Engineering-Theory \& Practice, 27(4): 126-133, (2007).

[7] Wei, G.W., Wang, X.R., "Some geometric aggregation operators based on interval-valued intuitionistic fuzzy sets and their application to group decision making", In Proceedings of the international conference on computational intelligence and security, 495-499, (2007).

[8] Wang, W., Liu, X., “ Interval-valued intuitionistic fuzzy hybrid weighted averaging operator based on Einstein operation and its application to decision making", Journal of Intelligent and Fuzzy Systems, 25(2): 279-290, (2013).

[9] Bhowmik, M., Pal, M., "Partition of generalized interval-valued intuitionistic fuzzy sets and some properties", International Journal of Applied Mathematical Analysis and Applications, 4(1): 1-10, (2009).

[10] Bhowmik, M., Pal, M., "Generalized interval-valued intuitionistic fuzzy sets", The Journal of Fuzzy Mathematics, 18(2): 357-371, (2010).

[11] Bhowmik, M., Pal, M., "Some results on generalized interval-valued intuitionistic fuzzy sets", International Journal of Fuzzy Systems, 14(2): 193-203, (2012).

[12] Li, D.F., "Linear programming method for MADM with interval-valued intuitionistic fuzzy sets", Expert Systems with Applications, 37(8): 5939-5945, (2010).

[13] Li, D.F., "TOPSIS-based nonlinear-programming methodology for multiattribute decision making with interval-valued intuitionistic fuzzy sets”, IEEE Transactions on Fuzzy Systems, 18(2): 299-311, (2010).

[14] Li, D.F., "Closeness coefficient based nonlinear programming method for interval-valued intuitionistic fuzzy multiattribute decision making with incomplete preference information", Applied Soft Computing, 11(4): 3402-3418, (2011).

[15] Yue, Z., "An approach to aggregating interval numbers into interval-valued intuitionistic fuzzy information for group decision making”, Expert Systems with Applications, 38(5): 6333-6338, (2011). 
[16] Chen, S.M., Yang, M.W., Yang, S.W., Sheu, T.W., Liau, C.J., "Multicriteria fuzzy decision making based on interval-valued intuitionistic fuzzy sets", Expert Systems with Applications, 39(15): 1208512091, (2012).

[17] Bai, Z., "An interval-valued intuitionistic fuzzy TOPSIS method based on an improved score function", The Scientific World Journal, Article ID 879089, 2013: 1-6, (2013).

[18] Wang, C.Y., Chen, S.M., "Multiple attribute decision making based on interval-valued intuitionistic fuzzy sets, linear programming methodology and the extended TOPSIS method", Information Sciences, 397-398: 155-167, (2017).

[19] Mondal, T.K., Samanta, S.K., "Topology of interval-valued intuitionistic fuzzy sets", Fuzzy Sets and Systems, 119(3): 483-494, (2001).

[20] Zhang, Z., Yang, J., Ye, Y., Zhang, Q.S., "A generalized interval valued intuitionistic fuzzy sets theory", Procedia Engineering, 15: 2037-2041, (2011).

[21] Sudharsan, S., Ezhilmaran, D., "Two new operator defined over interval valued intuitionistic fuzzy sets", International Journal of Fuzzy Logic Systems, 4(4): 1-13, (2014).

[22] Ananthi, V.P., Balasubramaniam, P., "Image fusion using interval-valued intuitionistic fuzzy sets", International Journal Image Data Fusion, 6: 249-269, (2015).

[23] Meng, F., Tan, C., Chen, X., “An approach to Atanassov's interval-valued intuitionistic fuzzy multiattribute decision making based on prospect theory", International Journal of Computational Intelligence Systems, 8(3): 591-605, (2015).

[24] Reiser, R.H.S., Bedregal, B., "Correlation in interval-valued Atanassov's intuitionistic fuzzy sets conjugate and negation operators", International Journal of Uncertainty, Fuzziness and KnowledgeBased Systems, 25(5): 787-819, (2017).

[25] Ahn, J.Y., Han, K.S., Oh, S.Y., Lee, C.D., “An application of interval-valued intuitionistic fuzzy sets for medical diagnosis of headache", International Journal of Innovative Computing, Information and Control, 7(5): 2755-2762, (2011).

[26] Ezhilmaran, D., Sudharsan, S., "Application of generalized interval valued intuitionistic fuzzy relation with fuzzy max-min composition technique in medical diagnosis", Applied Mathematical Sciences, 8(141): 7031-7038, (2014).

[27] Qi, X.W., Liang, C.Y., Zhang J., "Some generalized dependent aggregation operators with intervalvalued intuitionistic fuzzy information and their application to exploitation investment evaluation", Journal of Applied Mathematics, Article ID 705159, 24. doi:10.1155/2013/705159, 2013: 1-24, (2013).

[28] Wang, L.L., Li, D.F., Zhang, S.S., "Mathematical programming methodology for multiattribute decision making using interval-valued intuitionistic fuzzy sets", Journal of Intelligent \& Fuzzy Systems, 24(4): 755-763, (2013).

[29] Zhang, S., Yu, D., Wang, Y., Zhang, W., "Evaluation about the performance of E-government based on interval-valued intuitionistic fuzzy set", The Scientific World Journal, Article ID 234241, doi:10.1155/2014/234241, 2014: 1-10, (2014).

[30] Tan, C., Ma, B., Wu, D.D., Chen, X., "Multi-criteria decision making methods based on interval-valued intuitionistic fuzzy sets", International Journal of Uncertainty, Fuzziness and Knowledge-Based Systems, 22(03): 469-488, (2014). 
[31] Makui, A., Gholamian, M.R., Mohammadi, S.E., "Supplier selection with multi criteria group decision making based on interval valued intuitionistic fuzzy sets", Journal of Industrial and Systems Engineering, 8(4): 18-37, (2015).

[32] Pathinathan, T., Jon Arockiaraj, J., Ilavarasi, P., "An application of interval valued intuitionistic fuzzy sets in medical diagnosis using logical operators", International Journal of Computing Algorithm, 3(1): 495-49, (2014).

[33] Zhou, F., "Enterprise E-marketing performance evaluation based on interval-valued intuitionistic fuzzy sets OWA operator", Chemical Engineering Transactions, 46: 637-642, (2015).

[34] Baloui Jamkhaneh, E., Nadarajah, S., "A new generalized intuitionistic fuzzy sets”, Hacettepe Journal of Mathematics and Statistics, 44 (6): 1537-1551, (2015).

[35] Baloui Jamkhaneh, E., "The operators over the generalized intuitionistic fuzzy sets", International Journal of Nonlinear Analysis and Applications, 8(1): 11-21, (2017).

[36] Baloui Jamkhaneh, E., Nadi Ghara, A., "Four new operators over generalized intuitionistic fuzzy sets", Journal of New Theory, 18: 12-21, (2017).

[37] Baloui Jamkhaneh, E., Nadarajah, S., "On modal operators over generalized intuitionistic fuzzy set", Gazi University Journal of Science, 31(1): 222-234, (2018).

[38] Baloui Jamkhaneh, E., Garg, H., "Some new operations over the generalized intuitionistic fuzzy sets and their application to decision making process", Granular Computing, 3(2): 111-122, (2018).

[39] Baloui Jamkhaneh, E., "New generalized interval value intuitionistic fuzzy sets", Research and Communications in Mathematics and Mathematical Sciences, 5(1): 33-46, (2015).

[40] Baloui Jamkhaneh, E., "New operations over generalized interval valued intuitionistic fuzzy sets", Gazi University Journal of Science, 29(3): 667-674, (2016).

[41] Baloui Jamkhaneh, E., "On the modal operators over the generalized interval valued intuitionistic fuzzy sets", Journal of Applied Mathematics and Informatics, 35(5-6): 459-476, (2017).

[42] Baloui Jamkhaneh, E., Amirzadi, A., "New operators over the generalized interval valued intuitionistic fuzzy sets", Research and Communications in Mathematics and Mathematical Sciences, 8(2): 81-94, (2017). 